\title{
Conformable Derivatives in Laplace Equation and Fractional Fourier Series Solution
}

\author{
Ronak Pashaei ${ }^{1}$, Mohammad Sadegh Asgari ${ }^{1}$, Amir Pishkoo ${ }^{2 *}$ \\ ${ }^{1}$ Islamic Azad University, Central Tehran Branch, Tehran, Iran \\ ${ }^{2}$ Physics and Accelerators Research School, Nuclear Science and Technology Research Institute, \\ Tehran, Iran.
}

* Corresponding author email: apishkoo@gmail.com
Received: 10 August 2019 / Revised: 12 October 2019 / Accepted: 06 November 2019 / Published: 07 November 2019

\begin{abstract}
In this paper the solution of conformable Laplace equation, $\frac{\partial^{\alpha} u(x, y)}{\partial x^{\alpha}}+\frac{\partial^{\alpha} u(x, y)}{\partial y^{\alpha}}=$ 0 , where $1<\alpha \leq 2$ has been deduced by using fractional fourier series and separation of variables method. For special cases $\alpha=2$ (Laplace's equation), $\alpha=1.9$, and $\alpha=1.8$ conformable fractional fourier coefficients have been calculated. To calculate coefficients, integrals are of type "conformable fractional integral".
\end{abstract}

Keywords: Conformable fourier series, conformable derivative, conformable Laplace equation.

\section{Introduction}

History of "conformable" fractional derivative which is not really fractional derivative, based on limit definition, returns to five years ago (in 2014). However, about starting point of fractional derivative, it should be said that L'Hopital asked the question "What does derivative of order $\frac{1}{2}$ mean? namely $\frac{d^{\frac{1}{2}} f}{d x^{\frac{1}{2}}}$, in 1695. Many researchers have been trying to generalize the concept of an ordinary derivative to fractional derivative. Most of definitions use an integral form such as Riemann - Liouville definition or Caputo definition. However, Khalil et al. introduces the limit form for fractional derivative entitled "conformable fractional derivative" $[1-5]$.

Definition 1.1. [1] Given a function $f:[0, \infty) \rightarrow \mathbb{R}$

$$
T_{\alpha}(f)(t)=\lim _{\epsilon \rightarrow 0} \frac{f\left(t+\epsilon t^{1-\alpha}\right)-f(t)}{\epsilon} .
$$

for all $t>0, \alpha \in(0,1)$. If $f$ is $\alpha$-differentiable in some $(0, a), a>0$, and $\lim _{t \rightarrow 0^{+}} f^{(\alpha)}(t)$ exist, then define $f^{(\alpha)}(0)=\lim _{t \rightarrow 0^{+}} f^{(\alpha)}(t)$.

Khali et al. gives also via fractional cords a geometrical meaning to conformable fractional derivative [6]. In [7] Tarasov 
proves that Conformable derivatives can not be considered as fractional derivatives of non-integer order. Thus, it is better that instead of "Conformable fractional derivative" we just call it "Conformable derivative".

For a more general case $\alpha \in(n, n+1]$, and a natural number $n$ we have the following definition:

Definition 1.2. [1] Let $\alpha \in(n, n+1]$, and $f$ be an $n$-differentiable at $t$, where $t>0$. Then the conformable fractional derivative of $f$ of order $\alpha$ is defined as

$$
\begin{aligned}
& T_{\alpha}(f)(t)= \\
& \lim _{\epsilon \rightarrow 0} \frac{f^{([\alpha]-1)}\left(t+\epsilon t^{([\alpha]-\alpha)}\right)-f^{([\alpha]-1)}(t)}{\epsilon}
\end{aligned}
$$

where $[\alpha]$ is the smallest integer greater than or equal to $\alpha$.

The conformable fractional derivative $T_{\alpha}$ satisfies all the properties in the following theorem.

Theorem 1.1. [1,5] Let $\alpha \in(0,1]$ and $f, g$ be $\alpha$-differentiable at a point $t>0$. Then,

1. $T_{\alpha}[a f+b g]=a T_{\alpha}(f)+$ $b T_{\alpha}(g)$, for all $a, b \in \mathbb{R}$.

2. $T_{\alpha}\left(t^{p}\right)=p t^{p-\alpha}$ for all $p \in \mathbb{R}$.

3. $T_{\alpha}(\lambda)=0$, for all constant functions, $f(t)=\lambda$.

4. $T_{\alpha}(f g)=f T_{\alpha}(g)+g T_{\alpha}(f)$.

5. $T_{\alpha}\left(\frac{f}{g}\right)=\frac{g T_{\alpha}(f)-f T_{\alpha}(g)}{g^{2}}$.

6. If, in addition, $f$ is differentiable, then $T_{\alpha}(f)(t)=t^{1-\alpha} \frac{d f}{d t}(t)$.
Definition 1.3. [1] The $\alpha$-fractional integral of a function $f$ starting from $a \geq 0$ is defined as

$$
I_{\alpha}^{a}(f)(t)=I_{\alpha}^{a}\left(t^{\alpha-1} f\right)=\int_{a}^{t} \frac{f(x)}{x^{1-\alpha}} d x
$$

where the integral is the usual Riemann improper integral, and $\alpha \in(0,1)$.

Following the definition of conformable derivative and conformable integral, conformable differential equations, conformable integral equations and differential-integral equations involving them are also discussed. The conformable heat fractional partial differential equation and conformable diffusion fractional partial differential equation have been solved in $[8,9]$. Furthermore, in generalized format a generalization of classical Sturm-Lioville equation to conformable fractional Sturm-Lioville equation has been introduced by Bilender et al. [10]. The methods for solving these equations can be analytical, semi-analytical or numerical methods [11-17]. The Fourier series is one of the most important methods that are used in engineering and physical sciences to give analytic solution involving initial and boundary values. The method of fractional series is introduced by Abu Hammed and Khalil [2]. By using of two methods, including combining conformable fourier series and separation of variables, in this paper we solve conformable Laplace fractional partial differential equation. 


\section{Results and Discussion}

In order to solve conformable fractional Laplace's equation by method of fractional Fourier series we first need to consider conformable Fourier coefficients which can be calculated by using definition of conformable fractional integral. Calculation of conformable Fourier series coefficients are somewhat more complex than ordinary Fourier series. This series can be written as follows:

$$
\begin{aligned}
& S(f)(x)= \\
& \frac{a_{0}}{2}+\sum_{n=1}^{\infty}\left[a_{n} \cos \left(n \frac{x^{\alpha}}{\alpha}\right)+b_{n} \sin \left(n \frac{x^{\alpha}}{\alpha}\right)\right],
\end{aligned}
$$

where $a_{0}, a_{n}$, and $b_{n}$ are fractional Fourier coefficients.

$$
\begin{aligned}
a_{0} & =\left.\frac{1}{p} I_{\alpha}^{a}(f)(t)\right|_{a=0} ^{t=p=(\alpha 2 \pi)^{\frac{1}{\alpha}}} \\
& =\frac{1}{(\alpha 2 \pi)^{\frac{1}{\alpha}}} \int_{0}^{(\alpha 2 \pi)^{\frac{1}{\alpha}}} f(t) \frac{d t}{t^{1-\alpha}}(4)
\end{aligned}
$$

$$
\begin{gathered}
a_{n}=\left.\frac{2}{p} I_{\alpha}^{a}\left(f \cos n \frac{t^{\alpha}}{\alpha}\right)(t)\right|_{a=0} ^{t=p=(\alpha 2 \pi)^{\frac{1}{\alpha}}}(5) \\
=\frac{2}{(\alpha 2 \pi)^{\frac{1}{\alpha}}} \int_{0}^{(\alpha 2 \pi)^{\frac{1}{\alpha}}} f(t) \cos \left(n \frac{t^{\alpha}}{\alpha}\right) \frac{d t}{t^{1-\alpha}},(6) \\
n=1,2,3, \ldots \text { and } \\
b_{n}=\left.\frac{2}{p} I_{\alpha}^{a}\left(f \sin n \frac{t^{\alpha}}{\alpha}\right)(t)\right|_{a=0} ^{t=p=(\alpha 2 \pi)^{\frac{1}{\alpha}}}(7) \\
=\frac{2}{(\alpha 2 \pi)^{\frac{1}{\alpha}}} \int_{0}^{(\alpha 2 \pi)^{\frac{1}{\alpha}}} f(t) \sin \left(n \frac{t^{\alpha}}{\alpha}\right) \frac{d t}{t^{1-\alpha}},(8) \\
n=1,2,3, \ldots
\end{gathered}
$$

The definition of periodic function also differs from the ordinary case as follows:

Definition 2.1. [2] A function $f(t)$ is called $\alpha$-periodical with period $p$ if

$$
f(t)=g(\varphi(t))=g\left(\varphi(t)+\frac{p^{\alpha}}{\alpha}\right),
$$

for all $t \in[0, \infty)$.

Example 2.1. The function $f(t)=$ $\cos \left(\frac{t^{\alpha}}{\alpha}\right)$ is $\alpha$-periodic with period $(\alpha 2 \pi)^{\frac{1}{\alpha}}$. For value of $\alpha=\frac{2}{3}$, the function with period $\left(\frac{4 \pi}{3}\right)^{\frac{3}{2}}$ has been plotted in Figure 1. .

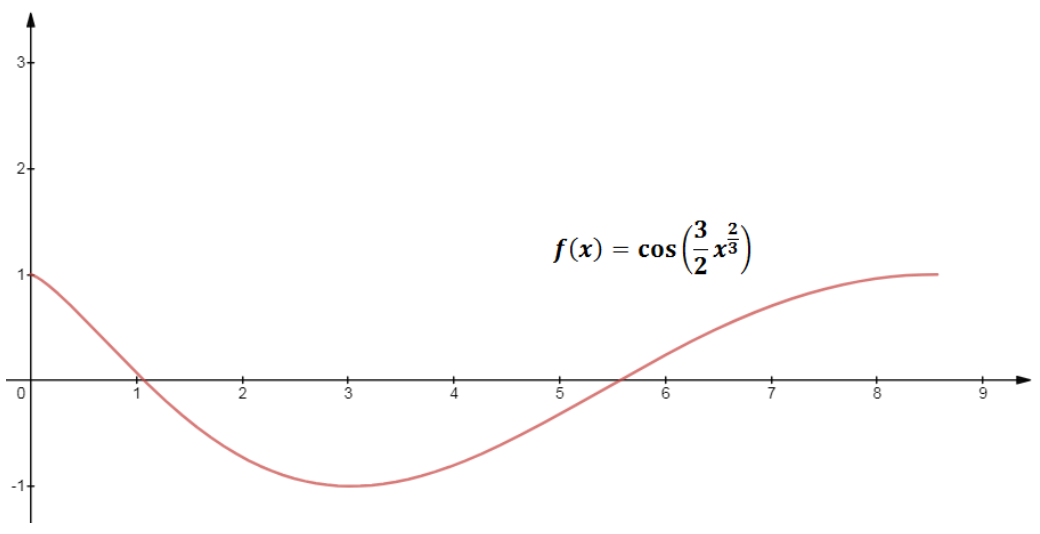

Figure 1: The function $f(x)=\cos \left(\frac{x^{\frac{2}{3}}}{\frac{2}{3}}\right)$ is $\frac{2}{3}$-periodic with period $\left(\frac{4 \pi}{3}\right)^{\frac{3}{2}}=8.573$. 
Now we apply such series to solve the following conformable fractional Laplace equation after using separation of variables method:

$$
\frac{\partial^{\alpha} u(x, y)}{\partial x^{\alpha}}+\frac{\partial^{\beta} u(x, y)}{\partial y^{\beta}}=0,
$$

where $1<\alpha \leq 2$ and $1<\beta \leq 2 ; \alpha=\beta$ along with the following boundary conditions:

$$
\begin{aligned}
& u(0, y)=u_{1}, u(L, y)=u_{2}, \\
& u(x, 0)=u_{3}, u(x, L)=u_{4} .
\end{aligned}
$$

We use the separation of variables method $(\mathrm{SVM})$ so that $u(x, y)=X(x) Y(y)$. Putting it in the (9) gives

$$
\frac{\partial^{\alpha} X(x)}{\partial x^{\alpha}} Y(y)+X(x) \frac{\partial^{\beta} Y(y)}{\partial x^{\beta}}=0
$$

or

$$
\frac{\frac{\partial^{\alpha} X(x)}{\partial x^{\alpha}}}{X(x)}+\frac{\frac{\partial^{\alpha} Y(y)}{\partial x^{\alpha}}}{Y(y)}=0
$$

Since $x$ and $y$ are independent variables, then we should have

$$
\frac{\frac{\partial^{\alpha} X(x)}{\partial x^{\alpha}}}{X(x)}=c, \frac{\frac{\partial^{\alpha} Y(y)}{\partial x^{\alpha}}}{Y(y)}=-c,
$$

where $c$ is constant and should be determined.

So we get the solution

$$
u(x, y)=e^{c\left(\frac{x^{\alpha}}{\alpha}-\frac{y^{\alpha}}{\alpha}\right)} .
$$

We can obtain the solution in terms of a double fourier series.

$$
\begin{aligned}
& S(X)(x)= \\
& \frac{a_{0}}{2}+\sum_{n=1}^{\infty}\left[a_{n} \cos \left(n \frac{x^{\alpha}}{\alpha}\right)+b_{n} \sin \left(n \frac{x^{\alpha}}{\alpha}\right)\right],
\end{aligned}
$$

$$
\begin{aligned}
& S(Y)(y)= \\
& \frac{c_{0}}{2}+\sum_{m=1}^{\infty}\left[c_{m} \cos \left(m \frac{y^{\alpha}}{\alpha}\right)+d_{n} \sin \left(m \frac{y^{\alpha}}{\alpha}\right)\right],
\end{aligned}
$$

$$
a_{0}=\frac{1}{(\alpha 2 \pi)^{\frac{1}{\alpha}}} \int_{0}^{(\alpha 2 \pi)^{\frac{1}{\alpha}}} e^{c \frac{x^{\alpha}}{\alpha}} \frac{d x}{x^{1-\alpha}}
$$

$$
a_{n}=\frac{2}{(\alpha 2 \pi)^{\frac{1}{\alpha}}} \int_{0}^{(\alpha 2 \pi)^{\frac{1}{\alpha}}} e^{c \frac{x^{\alpha}}{\alpha}} \cos \left(n \frac{x^{\alpha}}{\alpha}\right) \frac{d x}{x^{1-\alpha}},
$$$$
n=1,2,3, \ldots
$$

$$
\begin{aligned}
b_{n} & =\frac{2}{(\alpha 2 \pi)^{\frac{1}{\alpha}}} \int_{0}^{(\alpha 2 \pi)^{\frac{1}{\alpha}}} e^{e^{\frac{x^{\alpha}}{\alpha}}} \sin \left(n \frac{x^{\alpha}}{\alpha}\right) \frac{d x}{x^{1-\alpha}} \\
n & =1,2,3, \ldots
\end{aligned}
$$

And similarly we have

$$
c_{0}=\frac{1}{(\alpha 2 \pi)^{\frac{1}{\alpha}}} \int_{0}^{(\alpha 2 \pi)^{\frac{1}{\alpha}}} e^{-c \frac{y^{\alpha}}{\alpha}} \frac{d y}{y^{1-\alpha}}
$$

$$
\begin{gathered}
c_{m}=\frac{2}{(\alpha 2 \pi)^{\frac{1}{\alpha}}} \int_{0}^{(\alpha 2 \pi)^{\frac{1}{\alpha}}} e^{-c \frac{y^{\alpha}}{\alpha}} \cos \left(n \frac{y^{\alpha}}{\alpha}\right) \frac{d y}{y^{1-\alpha}}, \\
m=1,2,3, \ldots
\end{gathered}
$$

$$
\begin{gathered}
d_{n}=\frac{2}{(\alpha 2 \pi)^{\frac{1}{\alpha}}} \int_{0}^{(\alpha 2 \pi)^{\frac{1}{\alpha}}} e^{-c \frac{y^{\alpha}}{\alpha}} \sin \left(n \frac{y^{\alpha}}{\alpha}\right) \frac{d y}{y^{1-\alpha}}, \\
m=1,2,3, \ldots
\end{gathered}
$$

We have calculated above coefficients for the special cases standard Laplace's equation(namely $\alpha=\beta=2$ ), and conformable Laplace'equation (Laplace-like equation) for values $\alpha=1.9$ and $\alpha=1.8$ in Appendix section. 


\section{Conclusion}

This paper solves conformable Laplace equation, $\frac{\partial^{\alpha} u(x, y)}{\partial x^{\alpha}}+\frac{\partial^{\alpha} u(x, y)}{\partial y^{\alpha}}=0$, by combination of fractional fourier series and separation of variables method. Fractional Fourier coefficients are of type conformable fractional integral that can be calculated in MAPLE software. To solve Conformable wave-like equation, $\frac{\partial^{\alpha} u(x, t)}{\partial x^{\alpha}}-\frac{1}{c^{2}} \frac{\partial^{\alpha} u(x, t)}{\partial t^{\alpha}}=0$, conformable diffusion equation, $\frac{\partial^{\alpha} u(x, t)}{\partial x^{\alpha}}-\frac{1}{k} \frac{\partial^{\beta} u(x, t)}{\partial t^{\beta}}=0$, in which $1<\alpha \leq 2$ and $0<\beta \leq 1$, one can follow this approach.

\section{Competing Interests}

The authors declare that there are no conflicts of interest regarding the publication of this paper.

\section{How to Cite this Article:}

R. Pashaei, M. Asgari, and A. Pishkoo, "Conformable Derivatives in Laplace Equation and Fractional Fourier Series Solution", Int. Ann. Sci., vol. 9, no. 1, pp. 1-7, Nov. 2019. doi:10.21467/ias.9.1.1-7

\section{References}

[1] R. Khalil, M. Al Horani, A. Yousef, M. Sababheh, "A new definition of fractional derivative", Journal of Computational and Applied Mathematics, Vol. 264, pp. 65-70, 2014, http://dx.doi.org/doi:10.1016/j.cam.2014.01.002.

[2] R. Khalil, I. Abu Hammad, "Fractional fourier series with applications", American Journal of Computational and Applied Mathematics, Vol. 4, Issue 6, pp. 187-191, 2014, http://dx.doi.org/doi:10.5923/j.ajcam.20140406.01.
[3] M. D. Ortigueira, "Fractional Calculus for Scientists and Engineers", Book: Springer Dordrecht Heidelberg London New York, 2011.

[4] M. D. Ortigueira, J. A. Tenreiro Machado, "What is a fractional derivative?", Journal of Computational Physics, Vol. 293, pp. 4-13, 2015, http://dx.doi.org/doi:10.1016/j.jcp.2014.07.019.

[5] Udita N. Katugampola, "A new fractional derivative with classical properties", arXiv preprint, arXiv:1410.6535 (2014). http://arxiv.org/abs/1410.6535v2

[6] R. Khalil, M. Al Horani, M. Abu Hammad, "Geometric meaning of conformable derivative via fractional cords", Journal of Mathematics and Computer Science, Vol. 19, Issue 4, pp. 241-245, 2019, http://dx.doi.org/doi:10.22436/jmcs.019.04.03.

[7] V. E. Tarasov, No nonlocality. No fractional derivative, Communications in Non- linear Science and Numerical Simulation, Vol. 62 pp. 157-163, 2018, http://dx.doi.org/doi:10.1016/j.cnsns.2018.02.019

[8] R. Khalil, H. Abu Shaab, "Solution of some conformable fractional differential equation", International Journal of Pure and Applied Mathematics, Vol. 103, Issue 4, pp. 667-673, 2015, http://dx.doi.org/10.12732/ijpam.v103i4.6.

[9] D. Avci, B. B. Iskender Eroglu, N. Ozdemir, "Conformable heat equation on a radial symmetric plate", Thermal Science, Vol. 21, Issue 2, pp. 819-826, 2017, http://dx.doi.org/doi:10.2298/tsci160427302a.

[10] B. P. Allahverdiev, H. Tuna, Y. Yalçinkaya, "Conformable fractional Sturm-Liouville equation", Math. Meth. Appl. Sci., pp. 1-19, 2019, https://doi.org/10.1002/mma.5595.

[11] A. Dixit, A. Ujlayan, "Analytical solution to linear conformable fractional partial differential equations", World Scientific News, Vol. 113, pp. 49-56, 2018, https://doi.org/10.2298/TSCI160427302A.

[12] D. Avci, B. B. Iskender Eroglu, N. Ozdemir, "The dirichlet problem of a conformable advection-diffusion equation", Thermal Science, Vol. 21, Issue 1A, pp. 9-18, 2017, https://doi.orgdoi:10.2298/TSCI160421235A. 
[13] D. Avci, B. B. Iskender Eroglu, N. Ozdemir, "Conformable fractional wave-like equation on a radial symmetric plate", A. Babiarz et al. (eds.), Theory and Applications of Non-integer Order Systems, Lecture Notes in Electrical Engineering, 8th Conference on Non-integer Order Calculus and Its Applications, Zakopane, Poland 407, https://doi.org/doi:10.1007/978-3-319-45474-0.

[14] H. Ç. Yaslan, F. Mutlu, "Numerical solution of the conformable differential equations via shifted Legendre polynomials", International Journal of Computer Mathematics, 2019, http://dx.doi.org/doi:10.1080/00207160.2019.1605059.

[15] E. Ünal, A. Gökdoğan, "Solution of conformable fractional ordinary differen- tial equations via differential transform method", Optik, Vol. 128, pp. 264-273, 2017, http://dx.doi.org/doi:10.1016/j.ijleo.2016.10.031.

[16] O. Acan, D. Baleanu, "A new numerical technique for solving fractional partial differential equations", Miskolc Mathematical Notes, Vol. 19, Issue 1, pp. 3-18, 2018, https://doi.org/doi:10.18514/MMN.2018.2291.

[17] O. Acan, O. Firat, Y. Keskin, "Conformable variational iteration method, conformable fractional reduced differential transform method and conformable homotopy analysis method for nonlinear fractional partial differential equations", Waves in Random and Complex Media, 2018, https://doi.org/doi:10.1080/17455030.2018.1502485.

\section{Appendix}

1. Conformable fractional Fourier coefficients for $\alpha=2$

$$
\begin{aligned}
& {\left[>\frac{1}{(4 \pi)^{\frac{1}{2}}} \int_{0}^{(4 \pi)^{\frac{1}{2}}} x \mathrm{e}^{c \frac{x^{2}}{2}} \mathrm{dx}\right.} \\
& \boldsymbol{a}_{0}=\frac{1}{4} \frac{\left(-1+\mathrm{e}^{2 \pi c}\right) \sqrt{4}}{c \sqrt{\pi}} \\
& >\frac{1}{(4 \pi)^{\frac{1}{2}}} \int_{0}^{(4 \pi)^{\frac{1}{2}}} x \cos \left(n \frac{x^{2}}{2}\right) \mathrm{e}^{c \frac{x^{2}}{2}} \mathrm{~d} x \\
& \boldsymbol{a}_{n}=\frac{1}{4} \frac{\left(-c+2 \mathrm{e}^{2 \pi c} c \cos (\pi n)^{2}-\mathrm{e}^{2 \pi c} c+2 \mathrm{e}^{2 \pi c} n \sin (\pi n) \cos (\pi n)\right) \sqrt{4}}{\left(c^{2}+n^{2}\right) \sqrt{\pi}} \\
& >\frac{1}{(4 \pi)^{\frac{1}{2}}} \int_{0}^{(4 \pi)^{\frac{1}{2}}} x \sin \left(n \frac{x^{2}}{2}\right) \mathrm{e}^{c \frac{x^{2}}{2}} \mathrm{~d} x, \\
& \boldsymbol{b}_{n}=\frac{1}{4} \frac{\left(n+\mathrm{e}^{2 \pi c} n-2 \mathrm{e}^{2 \pi c} n \cos (\pi n)^{2}+2 \mathrm{e}^{2 \pi c} c \sin (\pi n) \cos (\pi n)\right) \sqrt{4}}{\left(c^{2}+n^{2}\right) \sqrt{\pi}}
\end{aligned}
$$

Figure 2: Fourier coefficients for solution of Laplace's equation $\frac{\partial^{2} u(x, y)}{\partial x^{2}}+\frac{\partial^{2} u(x, y)}{\partial y^{2}}=0$. 
2. Conformable fractional Fourier coefficients for $\alpha=1.9$

$$
\begin{aligned}
& >\frac{1}{(3.8 \pi)^{\frac{1}{1.9}}} \int_{0}^{(3.8 \pi)^{\frac{1}{1.9}}} x^{2 \cdot 9} \mathrm{e}^{\frac{e^{1.9}}{1.9}} \mathrm{~d} x \\
& \boldsymbol{a}_{\mathbf{0}}=\frac{0.4952799403\left(-1 .+\left(\mathrm{e}^{11.93805209} c\right)^{\frac{1052631579}{2000000000}}\right)}{c \pi^{0.5263157895}} \\
& >\frac{1}{(3.8 \pi)^{\frac{1}{1.9}}} \int_{0}^{(3.8 \pi)^{\frac{1}{1.9}}} 2^{9} \cos \left(n \frac{x^{19}}{1.9}\right) e^{e^{\frac{12.9}{1.9}}} \mathrm{dx} \\
& \frac{1}{\left(c^{2}+t^{2}\right) \pi^{0.5263157895}}\left(0 . 4 9 5 2 7 9 9 4 0 3 \left(-1 . c+\left(\mathrm{e}^{11.93805209 c} c\right)^{\frac{1052631579}{2000000000}} c \cos (6.283185310 n)\right.\right. \\
& \left.+\left(\mathrm{e}^{11.93805209 c)^{\frac{1052631579}{2000000000}} n \sin (6.283185310 n)}\right)\right) \\
& >\frac{1}{(3.8 \pi)^{\frac{1}{1.9}}} \int_{0}^{(3.8 \pi)^{\frac{1}{1.9}}} x^{0.9} \sin \left(n \frac{x^{1.9}}{1.9}\right) \mathrm{e}^{c \frac{x^{1.9}}{1.9}} \mathrm{~d} x \\
& -\frac{1}{\left(c^{2}+t^{2}\right) \pi^{0.5263157895}}\left(0 . 4 9 5 2 7 9 9 4 0 3 \left(-1 . n+\left(\mathrm{e}^{11.93805209} c\right)^{\frac{1052631579}{2000000000}} n \cos (6.283185310 n)\right.\right. \\
& \text {-1. } \left.\left.\left(\mathrm{e}^{11.93805209 c}\right)^{\frac{1052631579}{2000000000}} c \sin (6.283185310 \mathrm{n})\right)\right)
\end{aligned}
$$

(4)

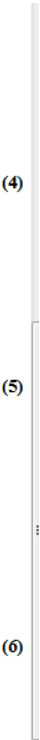

Figure 3: Fourier coefficients for solution of Laplace-like equation $\frac{\partial^{1.9} u(x, y)}{\partial x^{1.9}}+\frac{\partial^{1.9} u(x, y)}{\partial y^{1.9}}=$ 0 .

3. Conformable fractional Fourier coefficients for $\alpha=1.8$

$$
\begin{aligned}
& \mid>\frac{1}{(3.6 \pi)^{\frac{1}{1.8}}} \int_{0}^{(3.6 \pi)^{\frac{1}{1.8}}} 8^{0.8} \mathrm{e}^{\frac{c^{1.8}}{1.8}} \mathrm{dx} \\
& \boldsymbol{a}_{\mathbf{0}}=\frac{0.4908435123\left(-1 .+\left(\mathrm{e}^{11.30973355 c}\right)^{\frac{138888888899}{2500000000}}\right)}{c \pi^{5555555556}} \\
& \begin{array}{ll}
>\frac{1}{(3.6 \pi)^{\frac{1}{1.8}}} \int_{0}^{(3.6 \pi)^{\frac{1}{1.8}}} x^{0.8} \cos \left(n \frac{x^{1.8}}{1.8}\right) \mathrm{e}^{c \frac{c^{1.8}}{1.8}} \mathrm{dx} & \boldsymbol{a}_{\boldsymbol{n}}= \\
\frac{1}{\left(c^{2}+n^{2}\right) \pi^{0.5555555556}}\left(0 . 4 9 0 8 4 3 5 1 2 3 \left(-1 . c+\left(\mathrm{e}^{11.30973355 c}\right)^{\frac{13888888899}{2500000000}} c \cos (6.283185307 n)\right.\right.
\end{array} \\
& \left.\left.+\left(\mathrm{e}^{11.30973355 c}\right)^{\frac{1388888889}{2500000000}} n \sin (6.283185307 n)\right)\right) \\
& \begin{array}{l}
\frac{1}{(3.6 \pi)^{\frac{1}{1.8}}} \int_{0}^{(3.6 \pi)^{\frac{1}{1.8}}} x^{0.8} \sin \left(n \frac{x^{1.8}}{1.8}\right) \mathrm{e}^{c \frac{x^{1.8}}{1.8}} \mathrm{dx} \boldsymbol{b}_{\boldsymbol{n}}= \\
-\frac{1}{\left(c^{2}+n^{2}\right) \pi^{0.5555555556}}\left(0 . 4 9 0 8 4 3 5 1 2 3 \left(-1 . n+\left(\mathrm{e}^{11.30973355 c)^{\frac{1388888889}{2500000000}}} n \cos (6.283185307 n)\right.\right.\right. \\
\left.-1 .\left(\mathrm{e}^{11.30973355 c)^{\frac{1388888889}{2500000000}} c \sin (6.283185307 n)}\right)\right)
\end{array}
\end{aligned}
$$

Figure 4: Fourier coefficients for solution of Laplace-like equation $\frac{\partial^{1.8} u(x, y)}{\partial x^{1.8}}+\frac{\partial^{1.8} u(x, y)}{\partial y^{1.8}}=$ 0 . 